\title{
SERIES SOLUTION OF LARGE DEFORMATION OF A BEAM WITH ARBITRARY VARIABLE CROSS SECTION UNDER AN AXIAL LOAD
}

\author{
SHIJUN LIAO ${ }^{1}$ \\ (Received 16 November, 2008; revised 12 February, 2009)
}

\begin{abstract}
A general analytic approach is proposed for nonlinear eigenvalue problems governed by nonlinear differential equations with variable coefficients. This approach is based on the homotopy analysis method for strongly nonlinear problems. As an example, a beam with arbitrary variable cross section acted on by a compressive axial load is used to show its validity and effectiveness. This approach provides us with great freedom to transfer the original nonlinear buckling equation with variable coefficients into an infinite number of linear differential equations with constant coefficients that are much easier to solve. More importantly, it provides us with a convenient way to guarantee the convergence of solution series. As an example, the beam displacement and the critical buckling load can be obtained for arbitrary variable cross sections. The influence of nonuniformity of moment of inertia is investigated in detail and the optimal distributions of moment of inertia are studied. It is found that the critical buckling load of a beam with the optimal distribution of moment of inertia can be approximately $21-22 \%$ larger than that of a uniform beam with the same average moment of inertia. Mathematically, this approach is rather general and thus can be used to solve many other linear/nonlinear differential equations with variable coefficients.
\end{abstract}

2000 Mathematics subject classification: primary 47J10; secondary 34B15.

Keywords and phrases: eigenvalue, nonlinearity, variable coefficient, HAM, series, arbitrary beam.

\section{Introduction}

There are many interesting problems in engineering, such as nonlinear dynamics of beams with variable cross section [11, 16, 21, 24, 42], properties of structures made of functional graded materials $[13,17,18,20,36,43,45,50]$, the wave propagation in nonuniform waveguides [25], and the mechanical properties of nonuniform beams $[8-10,12,14,15,22,23,38]$. All of these problems are

\footnotetext{
${ }^{1}$ State Key Lab of Ocean Engineering, School of Naval Architecture, Ocean and Civil Engineering, Shanghai Jiao Tong University, Shanghai 200240, China; e-mail: sjliao@sjtu.edu.cn.

(C) Australian Mathematical Society 2010, Serial-fee code 1446-1811/2010 \$16.00
} 
governed by linear/nonlinear differential equations with variable coefficients, and have important practical applications. However, it is well known that nonlinear differential equations with variable coefficients are difficult to solve, especially by means of analytic techniques. The traditional analytic techniques such as perturbation methods cannot handle such problems effectively, because perturbation approximations are often strongly dependent upon small physical parameters and thus mostly valid only for problems with weak nonlinearity. Therefore, it is practically and theoretically meaningful to develop an effective analytic approach to solve this kind of nonlinear differential equation with variable coefficients.

Let us consider here a beam on two supports under a compressive axial load $P$ with arbitrary cross section, as shown in Figure 1. Let $l$ denote the length of such a beam, $\theta$ the rotation of the cross section and $u$ its deflection, respectively. Let $\xi$ denote the arc coordinate of the natural axis which passes through the centroid of each cross section of the beam in its straight or unbuckled state, $I(\xi)$ the smallest moment of inertia of the cross section about a line in its plane through the centroid, and $E$ Young's modulus of the beam material. Assume that all of the principle axes of inertia are parallel so that the beam is not twisted. However, unlike Keller [23], we consider here a more general case where the shape of each cross section does not need to be similar. Mathematically, the problem is governed by

$$
E I(\xi) \frac{d \theta}{d \xi}+P u=0, \quad u(0)=u(l)=0,
$$

where $I(\xi)$ is nonnegative. Differentiating the original governing equation (1.1) with respect to $\xi$ and using the relationship $\sin \theta=d u / d \xi$, we have the nonlinear buckling equation

$$
\left(E I \theta^{\prime}\right)^{\prime}+P \sin [\theta(\xi)]=0,
$$

where the prime denotes the differentiation with respect to $\xi$. Substituting $u(0)=$ $u(l)=0$ into (1.1) gives the corresponding boundary conditions $\theta^{\prime}(0)=\theta^{\prime}(l)=0$, which means that the bending moment at two ends of the beam is zero. The corresponding linear buckling equation is given by

$$
E I(\xi) u^{\prime \prime}(\xi)+P u(\xi)=0, \quad u(0)=u(l)=0 .
$$

The linear equation (1.3) has nonzero solutions only when the axial load is an eigenvalue, and the critical buckling load $P_{c}$ is given by the smallest eigenvalue. However, for the nonlinear buckling equation (1.2), nonzero solutions exist as long as the axial load $P$ is greater than the critical load $P_{c}$.

Define the dimensionless arc coordinate of the natural axis $s=(\xi / l) \pi$, and write $I=I_{0} \phi(\xi)=I_{0} \phi(s)$, where $\phi(s)$ is a distribution function of $I$, and $I_{0}$ is the average moment of inertia, defined by

$$
I_{0}=\frac{1}{l} \int_{0}^{l} I(\xi) d \xi=\frac{1}{\pi} \int_{0}^{\pi} I(s) d s .
$$




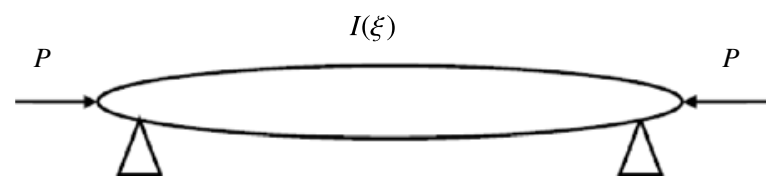

FIGURE 1. Beam acted on by a compressive axial load $P$ with variable moment of inertia $I(\xi)$.

Obviously,

$$
\frac{1}{\pi} \int_{0}^{\pi} \phi(s) d s=1 .
$$

The problem under consideration is governed by

$$
\phi(s) \theta^{\prime \prime}(s)+\phi^{\prime}(s) \theta^{\prime}(s)+\lambda \sin [\theta(s)]=0, \quad \theta^{\prime}(0)=\theta^{\prime}(\pi)=0,
$$

where the prime denotes differentiation with respect to $s$, and $\lambda=\left(P / E I_{0}\right)(l / \pi)^{2}$ is called the axial load parameter. As long as $\lambda$ is known, one has the axial load $P=\lambda\left(E I_{0}\right)(\pi / l)^{2}$. Note that $(1.5)$ is a second-order nonlinear ordinary differential equation (ODE) with two variable coefficients $\phi(s)$ and $\phi^{\prime}(s)$. Given an axial load $P$ and any a distribution $\phi(s)$ of the moment of inertia, we need to obtain convergent series of $\theta(s)$ by solving the above nonlinear ODE. This is not easy by means of the traditional analytic techniques. Keller [23] suggested an analytic approach based on perturbation methods for the nonlinear buckling equation (1.5), but without giving any results.

The homotopy analysis method (HAM) [27-31, 33, 35], an analytic method for highly nonlinear problems, has been widely applied to solve many nonlinear problems in science, engineering and finance $[1-7,26,37,40,41,44,46-49,51,52]$. Unlike perturbation techniques, the HAM is independent of any small physical parameters. More importantly, the HAM provides a simple way to ensure the convergence of solution series so that one can always get accurate enough approximations even for strongly nonlinear problems; see, for example, Abbasbandy [2] and Liang and Jeffrey [26]. Furthermore, the HAM provides great freedom to choose the so-called auxiliary linear operator so that one can approximate a nonlinear problem more effectively by means of better base functions, as illustrated by Liao and Tan [35]. This kind of freedom is so great that the second-order nonlinear two-dimensional Gelfand equation can be solved even by means of a fourth-order auxiliary linear operator in the frame of the HAM, as shown in [35]. In particular, by means of the HAM, a few new solutions of some nonlinear problems are found [32, 34] which are neglected by all other analytic methods and even by numerical techniques. All of these show the potential of the HAM. In this paper the HAM is applied to give a convergent series solution of the nonlinear differential equation (1.5) for any given distribution function $\phi(s)$ of the moment of inertia.

This paper is organized as follows. In Section 2 the basic idea of the analytic approach is described. In Section 3 the convergence of the solution series is 
investigated, and deformations of a beam with symmetrical or asymmetrical distributions of the moment of inertia $I$ studied. Further, the optimal distributions of $I$ are obtained for given average moment of inertia, $I_{0}$. Some conclusions are given in Section 4.

\section{Basic ideas}

In this section, Equation (1.5) is used as an example to describe the basic ideas of an analytic approach for nonlinear ODEs with variable coefficients. The homotopy analysis method [28, 30, 31, 35] is based on homotopy [19], a fundamental concept in topology and differential geometry which can be traced back to Poincaré [39]. In brief, by means of the HAM, one constructs a continuous mapping of an initial guess approximation to the exact solution of the equations considered. An auxiliary linear operator is chosen to construct such a continuous mapping, and an auxiliary parameter (called the convergence-control parameter) is used to ensure the convergence of the series solution. The method provides great freedom in choosing initial approximations and auxiliary linear operators. Through such freedom, a complicated nonlinear problem can be transformed into an infinite number of much simpler, linear subproblems, as shown by Liao and Tan [35].

There are two different strategies to solve the nonlinear eigenvalue problem under consideration. One is to regard the axial load parameter $\lambda$ as a given parameter, but consider the value of $\theta(s)$ at the boundary $s=0$ as unknown. The other is to regard $\lambda$ as unknown but consider $\theta(0)=\alpha$ as an additional boundary condition of $\theta(s)$, where $\alpha$ is given. Physically, these two strategies give the same result for the same axial load $P$ and the same distribution $\phi(s)$ of $I$. We adopt the second strategy: for the given distribution $\phi(s)$ of $I$ and the given value of $\alpha$ with the additional boundary condition $\theta(0)=\alpha$, our aim is to find the corresponding series solution of $\theta(s)$ and the unknown axial load parameter $\lambda$.

In this case, Equation (1.5) can be rewritten in the form

$$
\phi(s) \theta^{\prime \prime}(s)+\phi^{\prime}(s) \theta^{\prime}(s)+\lambda \sin [\theta(s)]=0, \quad 0<s<\pi,
$$

subject to the boundary conditions

$$
\theta^{\prime}(0)=\theta^{\prime}(\pi)=0, \quad \theta(0)=\alpha .
$$

For small $\theta$, one can linearize (2.1) as

$$
\phi(s) \theta^{\prime \prime}(s)+\phi^{\prime}(s) \theta^{\prime}(s)+\lambda \theta(s)=0, \quad 0<s<\pi .
$$

Even this linear ODE with variable coefficients is not easy to solve analytically.

Note that both of $\theta(s)$ and $\lambda$ are unknown for given $\alpha$. According to Equation (2.1), we define a nonlinear operator

$$
\begin{aligned}
& \mathcal{N}[W(s ; q), \Lambda(q), q] \\
& \quad=\phi(s) \frac{\partial^{2} W(s ; q)}{\partial s^{2}}+\phi^{\prime}(s) \frac{\partial W(s ; q)}{\partial s}+\Lambda(q)\left(\frac{\sin [q W(s ; q)]}{q}\right),
\end{aligned}
$$


where $q \in[0,1]$ is called the homotopy parameter, an embedding parameter without physical meaning, and $W(s ; q)$ and $\Lambda(q)$ correspond to the unknown $\theta(s)$ and $\lambda$, respectively. Note that $\sin [q W(s ; q)] / q$ corresponds to the term $\sin (\theta)$ in Equation (2.1), and tends to $W(s ; q)$ as $q \rightarrow 0$. Let $\theta_{0}(s)$, which satisfies the boundary conditions (2.2), denote an initial guess of $\theta(s)$. Let $\mathcal{L}$ denote an auxiliary linear operator with respect to $s$, and $\hbar$ a nonzero auxiliary parameter (called the convergence-control parameter), respectively. All of the initial guess $\theta_{0}(s)$, the auxiliary linear operator $\mathcal{L}$ and the convergence-control parameter $\hbar$ can be chosen with great freedom, as shown later. Then, we construct a two-parameter ${ }^{1}$ family of differential equations

$$
(1-q) \mathcal{L}\left[W(s ; q)-\theta_{0}(s)\right]=q \hbar \mathcal{N}[W(s ; q), \Gamma(q), q], \quad 0<s<\pi,
$$

subject to the boundary conditions

$$
\left.\frac{\partial W(s ; q)}{\partial s}\right|_{s=0}=\left.\frac{\partial W(s ; q)}{\partial s}\right|_{s=\pi}=0, \quad W(0 ; q)=\alpha .
$$

Obviously, when $q=0$, because $\mathcal{L}(0)=0$ for any linear operator $\mathcal{L}$, Equations (2.4) and (2.5) have the solution

$$
W(s ; 0)=\theta_{0}(s) .
$$

When $q=1$, since $\hbar \neq 0$, Equations (2.4) and (2.5) are equivalent to the original equations (2.1) and (2.2), provided that

$$
W(s ; 1)=\theta(s), \quad \Lambda(1)=\lambda .
$$

Write

$$
\Lambda(0)=\lambda_{0}
$$

where $\lambda_{0}$ denotes the initial guess of the axial load parameter $\lambda$. Then, as the homotopy parameter $q$ increases from 0 to $1, W(s ; q)$ varies (or deforms) from the initial guess $\theta_{0}(s)$ to the unknown exact solution $\theta(s)$, as does $\Lambda(q)$ from the initial guess $\lambda_{0}$ to the unknown $\lambda$. Obviously, the deformations of $W(s ; q)$ and $\Lambda(q)$ are completely determined by Equations (2.4) and (2.5), which are called the zeroth-order deformation equations.

On expanding $W(s ; q)$ and $\Lambda(q)$ into a Taylor series with respect to the homotopy parameter $q$, we have, using (2.6) and (2.8), the so-called homotopy series

$$
W(s ; q)=\theta_{0}(s)+\sum_{m=1}^{+\infty} \theta_{m} q^{m}, \quad \Lambda(q)=\lambda_{0}+\sum_{m=1}^{+\infty} \lambda_{m} q^{m}
$$

where

$$
\theta_{m}(s)=\mathcal{D}_{m}[W(s ; q)], \quad \lambda_{m}=\mathcal{D}_{m}[\Lambda(q)],
$$

1 They are the homotopy parameter $q \in[0,1]$ and the convergence-control parameter $\hbar$. 
with

$$
\mathcal{D}_{m} f:=\left.\frac{1}{m !} \frac{\partial^{m} f}{\partial q^{m}}\right|_{q=0} .
$$

Here, $\theta_{m}(s)$ and $\lambda_{m}$ are called the $m$ th-order homotopy derivative of $W(s ; q)$ and $\Lambda(q)$, respectively. We need to ensure that the convergence radius of the power series is finite. Fortunately, the homotopy series (2.9) contain the so-called convergence-control parameter $\hbar$, and besides we have great freedom to choose the auxiliary linear operator $\mathcal{L}$. Obviously, the convergence-control parameter $\hbar$ and the auxiliary linear operator $\mathcal{L}$ have great influence on the convergence of the homotopy series (2.9). Assume that the auxiliary linear operator $\mathcal{L}$ and the convergence-control parameter $\hbar$ are properly chosen so that the homotopy series (2.9) are convergent at $q=1$. Then, using (2.7), we have the so-called homotopy-series solution

$$
\theta(s)=\theta_{0}(s)+\sum_{m=1}^{+\infty} \theta_{m}, \quad \lambda=\lambda_{0}+\sum_{m=1}^{+\infty} \lambda_{m} .
$$

According to the fundamental theorems in calculus, each coefficient of the Taylor series of a smooth function is unique. Thus, $\theta_{m}(s)$ and $\lambda_{m}$ are unique, and are completely determined by $W(s ; q)$ and $\Lambda(q)$, respectively. Therefore, the governing equations and the boundary conditions of $\theta_{m}(s)$ and $\lambda_{m}$ can be directly deduced from the zeroth-order deformation equations (2.4) and (2.5). For simplicity, define the vectors

$$
\vec{\theta}_{n}=\left\{\theta_{0}(s), \theta_{1}(s), \theta_{2}(s), \ldots, \theta_{n}(s)\right\}, \quad \vec{\lambda}_{n}=\left\{\lambda_{0}, \lambda_{1}, \lambda_{2}, \ldots, \lambda_{n}\right\} .
$$

Differentiating the zeroth-order deformation equations (2.4) and (2.5) $m$ times with respect to $q$, then setting $q=0$, and finally dividing by $m$ !, we have the so-called mth-order deformation equation:

$$
\mathcal{L}\left[\theta_{m}(s)-\chi_{m} \theta_{m-1}(s)\right]=\hbar R_{m}\left(\vec{\theta}_{m-1}, \vec{\lambda}_{m-1}\right) \quad \text { where } \chi_{m}= \begin{cases}0, & m \leq 1 \\ 1, & m>1\end{cases}
$$

subject to the boundary conditions

$$
\theta_{m}^{\prime}(0)=\theta_{m}^{\prime}(\pi)=0, \quad \theta_{m}(0)=0,
$$

and

$$
\begin{aligned}
R_{m}\left(\vec{\theta}_{m-1}, \vec{\lambda}_{m-1}\right) & =\left.\frac{1}{(m-1) !} \frac{d^{m-1} \mathcal{N}[W(s ; q), \Gamma(q), q]}{d q^{m-1}}\right|_{q=0} \\
& =\phi(s) \theta_{m-1}^{\prime \prime}(s)+\phi^{\prime}(s) \theta_{m-1}^{\prime}(s)+\sum_{n=0}^{m-1} \lambda_{n} S_{m-n}(s)
\end{aligned}
$$


Note that, directly substituting (2.9) into the zeroth-order deformation equations (2.4) and (2.5), and then equating the coefficients of like powers of the homotopy parameter $q \in[0,1]$, one can get exactly the same high-order deformation equations mentioned above, as pointed out by Sajid et al. [40, 41]. A systematic method for obtaining highorder deformation equations in general cases is given by Liao [33]. Here, $S_{n}(s)$ is given by the recurrence formulas described in Appendix A. For example,

$$
\begin{aligned}
& R_{1}\left(\vec{\theta}_{0}, \vec{\lambda}_{0}\right)=\phi(s) \theta_{0}^{\prime \prime}(s)+\phi^{\prime}(s) \theta_{0}^{\prime}(s)+\lambda_{0} \theta_{0}(s), \\
& R_{2}\left(\vec{\theta}_{1}, \vec{\lambda}_{1}\right)=\phi(s) \theta_{1}^{\prime \prime}(s)+\phi^{\prime}(s) \theta_{1}^{\prime}(s)+\lambda_{0} \theta_{1}(s)+\lambda_{1} \theta_{0}(s), \\
& R_{3}\left(\vec{\theta}_{2}, \vec{\lambda}_{2}\right)=\phi(s) \theta_{2}^{\prime \prime}(s)+\phi^{\prime}(s) \theta_{2}^{\prime}(s)+\lambda_{0} \theta_{2}(s)+\lambda_{1} \theta_{1}(s)+\lambda_{2} \theta_{0}(s)-\frac{\lambda_{0}}{6} \theta_{0}^{3}(s),
\end{aligned}
$$

and so on. By means of symbolic computation software such as Mathematica, Maple or Matlab, it is not difficult to get $R_{m}\left(\vec{\theta}_{m-1}, \vec{\lambda}_{m-1}\right)$ for large $m$. Note that, if the linearized equation (2.3) is used, we have simply

$$
R_{m}\left(\vec{\theta}_{m-1}, \vec{\lambda}_{m-1}\right)=\phi(s) \theta_{m-1}^{\prime \prime}(s)+\phi^{\prime}(s) \theta_{m-1}^{\prime}(s)+\sum_{n=0}^{m-1} \lambda_{n} \theta_{m-1-n}(s) .
$$

Note also that $R_{m}\left(\vec{\theta}_{m-1}, \vec{\lambda}_{m-1}\right)$ is dependent only upon $\theta_{0}, \theta_{1}, \theta_{2}, \ldots, \theta_{m-1}$ and $\lambda_{0}$, $\lambda_{1}, \lambda_{2}, \ldots, \lambda_{m-1}$.

Note that Equation (2.12) is rather general, because the auxiliary linear operator $\mathcal{L}$, the initial guess $\theta_{0}(s)$ and the convergence-control parameter $\hbar$ in this equation are not yet determined. It should be emphasized that we have great freedom to choose $\theta_{0}(s)$ and $\mathcal{L}$. It is well known that, in the special case of $\phi(s)=1$, corresponding to a beam of uniform cross section, $\theta(s)$ can be expanded in a set of periodic base functions $\{\cos (n \kappa s) \mid n, \kappa \geq 1, n, \kappa \in \mathbf{N}\}$ as

$$
\theta(s)=\sum_{n=1}^{+\infty} b_{n} \cos (n \kappa s)
$$

where $\kappa \geq 1$ is a positive integer, called the mode-shape number. To include this special case and to satisfy the boundary condition (2.2), we choose the initial guess $\theta_{0}(s)=\alpha \cos (\kappa s)=\alpha\left(e^{\kappa s i}+e^{-\kappa s i}\right) / 2$, and the auxiliary linear operator

$$
\mathcal{L} \theta=\theta^{\prime \prime}+\kappa^{2} \theta
$$

with the property that $\mathcal{L}\left[C_{1} \cos (\kappa s)+C_{2} \sin (\kappa s)\right]=0$, where $C_{1}, C_{2}$ are constant coefficients and $i=\sqrt{-1}$ represents the imaginary unit. Note that the linear term $\phi(s) \theta^{\prime \prime}(s)+\phi^{\prime}(s) \theta^{\prime}(s)$ in Equation (2.1) contains two variable coefficients $\phi(s)$ and $\phi^{\prime}(s)$. It is much more difficult to solve the corresponding linearized differential equation with arbitrary variable coefficients

$$
\phi(s) \theta^{\prime \prime}(s)+\phi^{\prime}(s) \theta^{\prime}(s)+\lambda \theta(s)=0
$$


than the linear differential equation with constant coefficients

$$
\theta^{\prime \prime}(s)+\kappa^{2} \theta(s)=0
$$

so it is not a good idea to choose

$$
\mathcal{L} \theta=\phi(s) \theta^{\prime \prime}(s)+\phi^{\prime}(s) \theta^{\prime}(s)+\lambda \theta(s)
$$

as the auxiliary linear operator. Fortunately, in contrast to other analytic techniques, the HAM provides great freedom and flexibility to choose an auxiliary linear operator (2.14) such that the corresponding high-order deformation equation (2.12) is a linear ODE with known constant coefficients. So, by choosing this kind of auxiliary linear operator (2.14), it becomes much easier to solve the high-order deformation equations (2.12) and (2.13), so that we can get analytic results at a rather high order of approximation by means of symbolic computation software. In this way, we transfer the original nonlinear ODE with arbitrary variable coefficients into an infinite number of linear ODEs with the known constant coefficients. Such a transformation does not need any small physical parameters.

It is worthwhile to emphasize the great flexibility and freedom of the HAM in the choice of the auxiliary linear operator: for example, by means of the HAM, one can even transform the second-order nonlinear Gelfand equation into an infinite number of fourth-order (in the two-dimensional case) or sixth-order (in the three-dimensional case) linear partial differential equations so as to greatly simplify resolving the original problem, as illustrated by Liao and Tan [35]. Similarly, using the great freedom and flexibility of the HAM in the choice of the auxiliary linear operator, we transform the original nonlinear ODE (2.1) with arbitrary variable coefficients into an infinite number of linear ODEs (2.12) with the known constant coefficients. In this way, it becomes much easier to solve the original nonlinear problem.

As mentioned above, the $m$ th-order deformation equations (2.12) and (2.13) are linear ODEs with constant coefficients and thus are easy to solve. Note that Equation (2.12) contains two unknowns: $\theta_{m}(s)$ and $\lambda_{m-1}$. Let $\bar{\theta}_{m}(s)$ denote a special solution of Equation (2.12). Its general solution reads

$$
\theta_{m}(s)=\bar{\theta}_{m}(s)+\chi_{m} \theta_{m-1}(s)+C_{m} \cos (\kappa s)+D_{m} \sin (\kappa s),
$$

where $C_{m}$ and $D_{m}$ are integral coefficients to be determined. The boundary conditions $\theta_{m}^{\prime}(0)=0$ and $\theta_{m}^{\prime}(\pi)=0$ give us two algebraic equations

$$
\begin{gathered}
\bar{\theta}_{m}^{\prime}(0)+\chi_{m} \theta_{m-1}^{\prime}(0)+D_{m} \kappa=0, \\
\bar{\theta}_{m}^{\prime}(\pi)+\chi_{m} \theta_{m-1}^{\prime}(\pi)+D_{m} \kappa \cos (\kappa \pi)=0 .
\end{gathered}
$$

Equations (2.15) and (2.16) contain two unknowns: $D_{m}$ and $\lambda_{m-1}$. From (2.15),

$$
D_{m}=-\frac{\bar{\theta}_{m}^{\prime}(0)+\chi_{m} \theta_{m-1}^{\prime}(0)}{\kappa} .
$$


Substituting (2.17) into (2.16) gives

$$
\bar{\theta}_{m}^{\prime}(\pi)+\chi_{m} \theta_{m-1}^{\prime}(\pi)=\left[\bar{\theta}_{m}^{\prime}(0)+\chi_{m} \theta_{m-1}^{\prime}(0)\right] \cos (\kappa \pi) .
$$

Solving Equation (2.18) gives $\lambda_{m-1}$. Furthermore, using the boundary condition $\theta_{m}(0)=0$ leads to

$$
C_{m}=-\bar{\theta}_{m}(0)-\chi_{m} \theta_{m-1}(0)
$$

In this way, we obtain $\lambda_{0}, \theta_{1}(s)$ by solving the first-order deformation equations, then $\lambda_{1}, \theta_{2}(s)$ by the second-order deformation equations, and so on. This is easy to do by means of symbolic computation software. In this way we can get results to a high order of approximation. The $M$ th-order approximation is given by

$$
\theta(s) \approx \theta_{0}(s)+\sum_{n=1}^{M} \theta_{n}(s), \quad \lambda \approx \lambda_{0}+\sum_{n=1}^{M} \lambda_{n} .
$$

Note that to get this approximation we had to solve $(M+1)$ th-order deformation equations (2.12) and (2.13).

It should be emphasized that the above approach is valid for an arbitrary distribution function $\phi(s) \geq 0$ of the moment of inertia, as shown in the following section. Besides, the value of the convergence-control parameter $\hbar$ has not yet been chosen, which provides us with a convenient way to guarantee the convergence of the solution series, as shown later.

\section{Series solution}

Let $(x, u)$ denote the coordinates of a point on the beam, where $u(s)$ is the displacement. Then

$$
x(s)=\int_{0}^{s} \cos [\theta(s)] d s, \quad u(s)=\int_{0}^{s} \sin [\theta(s)] d s,
$$

where $0 \leq s \leq \pi$ is the arc length of the beam. If $\theta(s)$ is a solution when $\alpha=\gamma$, then $-\theta(s)$ is the solution when $\alpha=-\gamma$. So it is necessary only to discuss the case of $\alpha \geq 0$.

3.1. Results for a symmetrical distribution of $\boldsymbol{I}$ From a practical point of view, the distribution of the moment of inertia of a beam is usually symmetrical. For the sake of simplicity, let us first consider the case $\phi(s)=1+\beta \cos (2 s)$, where $|\beta|<1$ and $0 \leq s \leq \pi$. In this case, the distribution of $I$ is symmetrical with respect to the center of beam. The case $\beta=0$ corresponds to a beam with the same moment of inertia. When $\beta>0$, the distribution of $I$ is dumbbell-shaped: it has the maximum value $1+\beta$ at the ends of a beam and decreases monotonically to the minimum value $1-\beta$ at the center. When $\beta<0$, the distribution of $I$ is olive-shaped: it has maximum value $1+|\beta|$ at the center and decreases monotonically to the minimum value $1-|\beta|$ at the ends. Some examples of the symmetric distribution of the moment of inertia are shown in Figure 2. 


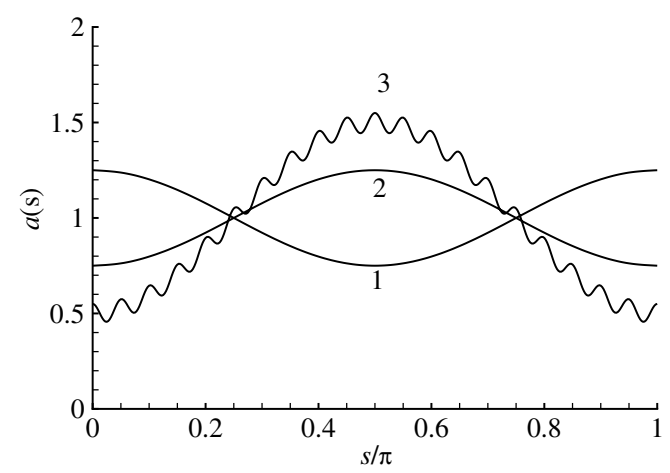

FIGURE 2. Examples of the distribution of the moment of inertia: curve 1, dumbbell-shaped distribution where $\phi(s)=1+\cos (2 s) / 4$; curve 2 , olive-shaped distribution where $\phi(s)=1-\cos (2 s) / 4$; curve 3 , olive-shaped distribution where $\phi(s)=1-\cos (2 s) / 2+\cos (40 s) / 20$.

It is well known that the deformation of a beam under axial load can have different patterns (or mode shapes), determined by different values of the mode-shape number $\kappa$. First of all, let us consider the case $\kappa=1$, which corresponds to the simplest mode shape of deformation. Note that our approximations contain the convergencecontrol parameter $\hbar$, which appears in the high-order deformation equation (2.12). For example, the first-, second- and third-order approximations of $\lambda$ read

$$
\begin{aligned}
\lambda \approx 1-\frac{\beta}{2}+\frac{9}{32} \beta^{2} \hbar, \\
\lambda \approx 1-\frac{\beta}{2}+\frac{9}{32} \beta^{2} \hbar(2+\hbar)+\frac{9}{512} \beta^{3} \hbar^{2}+\frac{1}{8}\left(1-\frac{\beta}{2}\right) \alpha^{2}, \\
\lambda \approx 1-\frac{\beta}{2}+\frac{9}{32} \beta^{2} \hbar\left(3+3 \hbar+\hbar^{2}\right)+\frac{9}{512} \beta^{3} \hbar^{2}(3+2 \hbar)+\frac{603}{8192} \beta^{4} \hbar^{3} \\
\quad+\frac{1}{8}\left[1-\frac{1}{8} \beta(4+\hbar)+\frac{11}{32} \beta^{2} \hbar\right] \alpha^{2},
\end{aligned}
$$

respectively. The corresponding first- and second-order approximations of $\theta(s)$ are

$$
\begin{aligned}
\theta(s) \approx & \alpha \cos (s)-\left(\frac{3}{16}\right) \alpha \beta \hbar[\cos (s)-\cos (3 s)] \\
\theta(s) \approx & \alpha \cos (s)-\left(\frac{3}{16}\right) \alpha \beta \hbar(2+\hbar)[\cos (s)-\cos (3 s)] \\
& -\left(\frac{3}{256}\right) \alpha \beta^{2} \hbar^{2}[3 \cos (s)+2 \cos (3 s)-5 \cos (5 s)]
\end{aligned}
$$

respectively.

As pointed out by Liao [30, 31, 35], the convergence-control parameter $\hbar$, an auxiliary parameter without physical meaning, provides a simple way to control and 
TABLE 1 . The value of $\lambda$ at different orders of approximation and values of $\hbar$ for the case where $\alpha=1$ and $\phi(s)=1-0.5 \cos (2 s)$.

\begin{tabular}{ccccc}
\hline & \multicolumn{4}{c}{ Calculated $\lambda$} \\
\cline { 2 - 5 } Order of approx. & $\hbar=-1.1$ & $\hbar=-1$ & $\hbar=-0.9$ & $\hbar=-0.8$ \\
\hline 2 & 1.3340 & 1.3337 & 1.3349 & 1.3374 \\
4 & 1.3236 & 1.3233 & 1.3238 & 1.3252 \\
6 & 1.3209 & 1.3207 & 1.3209 & 1.3214 \\
8 & 1.3204 & 1.3203 & 1.3204 & 1.3206 \\
10 & 1.3203 & 1.3203 & 1.3203 & 1.3204 \\
15,20 & 1.3203 & 1.3203 & 1.3203 & 1.3203 \\
\hline
\end{tabular}

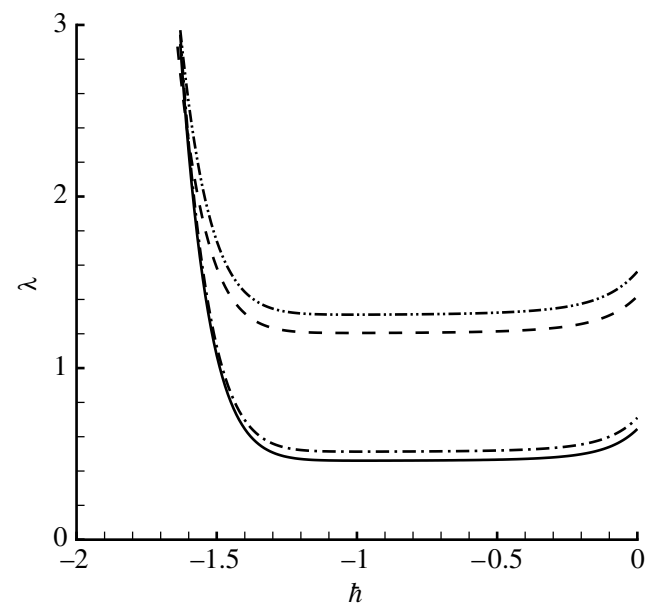

FIGURE 3. The curves of $\lambda \sim \hbar$ at the tenth-order of approximation in the case of $\phi(s)=1+\beta \cos (2 s)$ when $\kappa=1$. Solid line, $\alpha=1 / 2, \beta=3 / 4$; dashed line, $\alpha=1 / 2, \beta=-3 / 4$; dash-dotted line, $\alpha=1, \beta=$ 3/4; dash-dot-dotted line, $\alpha=1, \beta=-3 / 4$.

adjust the convergence of the HAM series. Mathematically, according to Liao's proof $[30,31,35]$ in the general case, the homotopy series $(2.11)$ must be the solution of the original nonlinear ODEs (2.1) and (2.2), as long as they are convergent. On the other hand, from a physical point of view, the value of $\lambda$ is completely determined for given $\alpha$ and $\beta$. Therefore, given $\alpha$ and $\beta$, all convergent homotopy-series solutions of $\lambda$ obtained by different values of $\hbar$ should give the same result. This is indeed true, as shown in Figure 3 for different values of $\alpha$ and $\beta$. Observing the curves $\lambda \sim \hbar$ in Figure 3, it is obvious that the series of $\lambda$ is convergent for all values of $\alpha$ and $\beta$ considered when $-5 / 4 \leq \hbar \leq-1 / 4$. For example, when $\alpha=1$ and $\beta= \pm 1 / 2$, the series of $\lambda$ given by different values of $\hbar \in[-5 / 4,-1 / 4]$, such as $\hbar=-1.1,-1,-0.9$ and -0.8 , converge to the same result $\lambda=1.3203$ for $\beta=-1 / 2$, and $\lambda=0.7764$ for $\beta=1 / 2$, respectively, as shown in Tables 1 and 2 . Further, it is found that, as long as 
TABLE 2. The value of $\lambda$ at different orders of approximation and values of $\hbar$ for the case $\alpha=1$ and $\phi(s)=1+0.5 \cos (2 s)$.

\begin{tabular}{ccccc}
\hline & \multicolumn{4}{c}{ Calculated $\lambda$} \\
\cline { 2 - 5 } Order of approx. & $\hbar=-1.1$ & $\hbar=-1$ & $\hbar=-0.9$ & $\hbar=-0.8$ \\
\hline 2 & 0.7768 & 0.7756 & 0.7759 & 0.7777 \\
4 & 0.7762 & 0.7758 & 0.7760 & 0.7768 \\
6 & 0.7763 & 0.7762 & 0.7763 & 0.7765 \\
$8,10,15,20$ & 0.7764 & 0.7764 & 0.7764 & 0.7764 \\
\hline
\end{tabular}

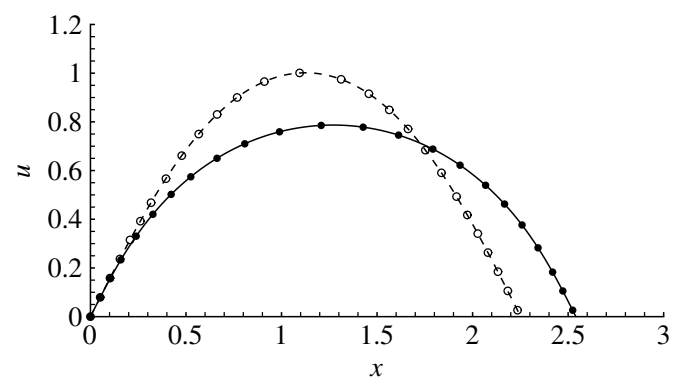

FIgURE 4. Comparison of the beam displacement at different orders of approximation for $\alpha=1$ and $\phi(s)=1+\beta \cos (2 s)$ by means of $\hbar=-1$. Solid line, fifth-order approximation when $\beta=-1 / 2$; dashed line, fifth-order approximation when $\beta=1 / 2$; filled circles, tenth-order approximation when $\beta=-1 / 2$; open circles, tenth-order approximation when $\beta=1 / 2$.

the homotopy-series solution of $\lambda$ is convergent, the corresponding homotopy-series solution of $\theta(s)$ is also convergent. For example, in case of $\alpha=1$ and $\beta= \pm 1 / 2$, the fifth-order approximation of the beam displacement agrees well with the tenth-order approximation, as shown in Figure 4. It is found that, when $-5 / 4 \leq \hbar \leq-1 / 4$, the homotopy-series solutions of $\theta(s)$ and $\lambda$ converge for all possible values of $\alpha$ and $\beta$ having physical meaning, and the tenth-order approximation is accurate enough. So, by means of the convergence-control parameter $\hbar$, we can always get convergent series solution of $\theta(s)$ and $\lambda$. This is an advantage of our approach over other analytic techniques.

For simplicity, we choose $\hbar=-1$ here. In the case of $\phi(s)=1+\beta \cos (2 s)$ and $\kappa=1$, it is useful to define

$$
\begin{aligned}
\lambda_{c}^{[10]}= & 1-\frac{\beta}{2}-\frac{9}{32} \beta^{2}+\frac{9}{512} \beta^{3}-\frac{603}{8192} \beta^{4}+\frac{1341}{131071} \beta^{5} \\
& -\frac{159687}{4194304} \beta^{6}+0.00713528 \beta^{7}-0.0244546 \beta^{8} \\
& +0.00544059 \beta^{9}-0.0175187 \beta^{10}+0.00437613 \beta^{11} .
\end{aligned}
$$


Now the corresponding tenth-order approximation of $\lambda$ reads

$$
\begin{aligned}
\lambda \approx & \lambda_{c}^{[10]}+\frac{1}{8}\left(1-0.375 \beta-0.351563 \beta^{2}+0.0246582 \beta^{3}-0.093277 \beta^{4}\right. \\
& +0.0143995 \beta^{5}-0.0486857 \beta^{6}+0.0100691 \beta^{7}-0.0314851 \beta^{8} \\
& \left.-0.00364847 \beta^{9}\right) \alpha^{2}+\left(0.0110677-0.00297038 \beta-0.00437249 \beta^{2}\right. \\
& +0.000240813 \beta^{3}-0.00115582 \beta^{4}+0.000141895 \beta^{5}-0.000665628 \beta^{6} \\
& \left.-0.000186064 \beta^{7}\right) \alpha^{4}+\left(9.16884-1.80011 \beta-3.74461 \beta^{2}+0.159778 \beta^{3}\right. \\
& \left.-1.25504 \beta^{4}-0.291921 \beta^{5}\right) 10^{-4} \alpha^{6} \\
& +\left(7.69883-1.07922 \beta-3.89926 \beta^{2}-0.3665482 \beta^{3}\right) 10^{-5} \alpha^{8} \\
& +(3.628201-1.814100 \beta) 10^{-6} \alpha^{10} .
\end{aligned}
$$

For given $\alpha$, the axial load parameter $\lambda$ has a maximum value in the region $\beta \in[-0.7,-0.6]$ and the minimum value at $\beta=1$, as shown in Figure 5. Thus, generally speaking, a beam with olive-shaped distribution of moment of inertia is safer than that with dumbbell-shaped ones. For given $\beta, \lambda$ has minimum value $\lambda_{c}$ as $\alpha \rightarrow 0$. Note that the beam has no displacement when $0 \leq \lambda \leq \lambda_{c}$, so $\lambda_{c}$ is regarded as the critical axial load value and thus has important physical significance. At the tenth-order of approximation, in the case of $\phi(s)=1+\beta \cos (2 s)$

$$
\lambda_{c} \approx \lambda_{c}^{[10]}
$$

where $\lambda_{c}^{[10]}$ is given by Equation (3.1). Formula (3.2) agrees well with the 40th-order approximation of $\lambda_{c}$, as shown in Figure 6 . At the tenth-order of approximation, $\lambda_{c}$ has the maximum value 1.1828 at $\beta=-0.633643$. At the 40th order of approximation, $\lambda_{c}$ has maximum value 1.1827 at $\beta=-0.63223$. The relative error is less than $0.01 \%$. Thus, (3.2) is quite accurate from a practical viewpoint. Given $\lambda_{c}$, it is easy to get the critical buckling load $P_{c}=\lambda_{c}\left(E I_{0}\right)(\pi / l)^{2}$. Note that a beam has no displacement if $P \leq P_{c}$.

Similarly, in the case of $\phi(s)=1+\beta \cos (2 s)$ with the mode-shape number $\kappa=2$, the tenth-order approximation of $\lambda_{c}$ with $\hbar=-1$ reads

$$
\begin{aligned}
\lambda_{c} \approx & 4-1.333333 \beta^{2}-0.351852 \beta^{4}-0.182922 \beta^{6} \\
& -0.117919 \beta^{8}-0.0847052 \beta^{10},
\end{aligned}
$$

which is always less than 4 , the value of $\lambda_{c}$ for a beam with uniform cross section when $\kappa=2$. In particular, for given $\beta, \lambda_{c}$ in the case of $\kappa=2$ is always greater than that for the case of $\kappa=1$, as shown in Figure 6. Similar phenomena are found for all $\kappa>1$. So, for a beam with arbitrary variable cross section, one need only consider $\lambda_{c}$ in the case of the mode-shape number $\kappa=1$. Thus, when $\phi(s)=1+\beta \cos (2 s)$, the optimal distribution of moment of inertia should be very close to $\phi(s)=1-0.63223 \cos (2 s)$. 


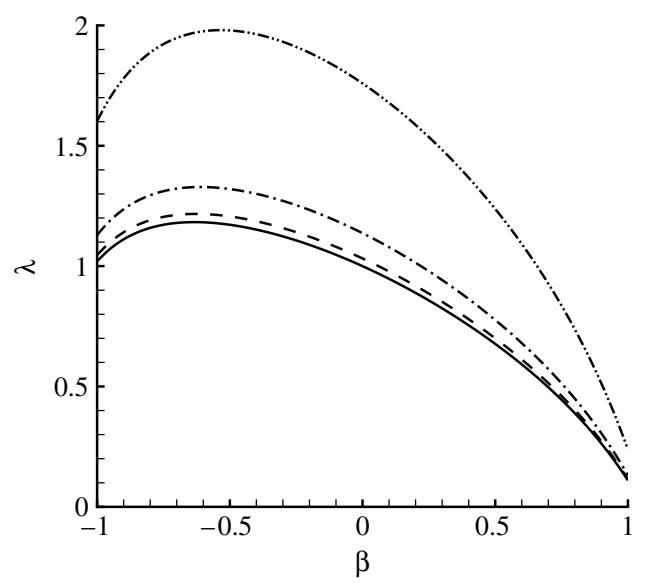

FIGURE 5. The curves of $\lambda$ versus $\beta$ at the tenth-order of approximation for $\phi(s)=1+\beta \cos (2 s)$ when $\kappa=1$. Solid line, $\alpha=0$; dashed line, $\alpha=1 / 2$; dash-dotted line, $\alpha=1$; dash-dot-dotted line, $\alpha=2$.

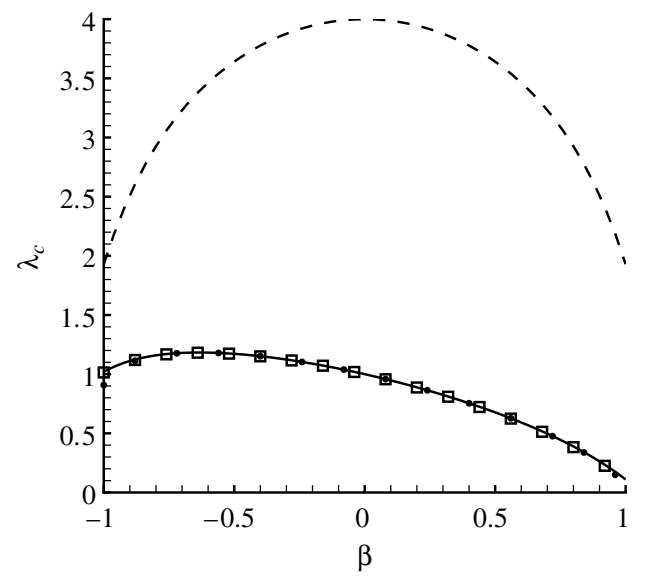

FIgURE 6. The critical axial load value $\lambda_{c}$ versus $\beta$ in the case of $\phi(s)=1+\beta \cos (2 s)$. Solid line, tenth-order approximation (6) when $\kappa=1$; circles, 40th-order approximation when $\kappa=1$; dashed line, tenth-order approximation when $\kappa=2$; square, tenth-order approximation (3.3) in the case of $\phi(s)=1+\beta \cos (2 s)+\cos (40 s) / 20$ when $\kappa=1$.

The above approach can be applied to get $\lambda_{c}$ for a more complicated distribution of moment of inertia. For example, when $\phi(s)=1+\beta \cos (2 s)+\cos (40 s) / 20$, the tenth-order approximation of $\lambda_{c}$ with $\kappa=1$ and $\hbar=-1$ reads

$$
\begin{aligned}
\lambda_{c} \approx & 0.998748-0.500627 \beta-0.282937 \beta^{2}+0.0168626 \beta^{3}-0.0756039 \beta^{4} \\
& +0.00945887 \beta^{5}-0.0403320 \beta^{6}+0.00632020 \beta^{7}-0.0269003 \beta^{8} \\
& +0.00462114 \beta^{9}-0.0175187 \beta^{10}+0.00437613 \beta^{11}
\end{aligned}
$$


The difference between (3.3) and $\lambda_{c}$ given by (3.2) for $\phi(s)=1+\beta \cos (2 s)$ is rather small, as shown in Figure 6. It indicates that the influence of small highfrequency terms in the distribution $\phi(s)$ of the moment of inertia on $\lambda_{c}$ can be neglected. Thus, the small inaccuracy of the moment of inertia of a beam caused by machine precision can be neglected.

As mentioned above, for $\phi(s)=1+\beta \cos (2 s), \lambda_{c}$ has maximum value $\left(\lambda_{c}\right)_{\max }=$ 1.183 at $\beta=-0.63223$, corresponding to the optimal distribution of the moment of inertia

$$
\phi^{*}(s)=1-0.63223 \cos (2 s) .
$$

In the general case $\phi(s)=1+\sum_{j=1}^{M} \beta_{j} \cos (2 j s)$, one can find the corresponding $\left(\lambda_{c}\right)_{\max }$ for given $M$ in a similar way. For example, for $\phi(s)=1+\beta_{1} \cos (2 s)+$ $\beta_{2} \cos (4 s)$, we have first- and second-order approximations (with $\hbar=-1$ ) of the critical axial load value

$$
\begin{aligned}
& \lambda_{c} \approx \lambda_{c}^{[1]} \equiv 1-\frac{\beta_{1}}{2}-\frac{9}{32} \beta_{1}^{2}+\frac{9}{16} \beta_{1} \beta_{2}-\frac{13}{24} \beta_{2}^{2}, \\
& \lambda_{c} \approx \lambda_{c}^{[2]} \equiv \lambda_{c}^{[1]}+\frac{9}{512} \beta_{1}^{3}+\frac{33}{128} \beta_{1}^{2} \beta_{2}-\frac{311}{1152} \beta_{1} \beta_{2}^{2} .
\end{aligned}
$$

At the tenth-order of approximation, $\lambda_{c}$ has the maximum value $\left(\lambda_{c}\right)_{\max }=1.206$ at $\beta_{1}=-0.6121$ and $\beta_{2}=-0.1957$, corresponding to the optimal distribution of moment of inertia

$$
\phi^{*}(s)=1-0.6121 \cos (2 s)-0.1957 \cos (4 s) .
$$

When $M=3, \lambda_{c}$ at the tenth-order of approximation has the maximum value $\left(\lambda_{c}\right)_{\max }=1.213$ at $\beta_{1}=-0.6193, \beta_{2}=-0.1719$ and $\beta_{3}=-0.1114$, corresponding to the optimal distribution

$$
\phi^{*}(s)=1-0.6193 \cos (2 s)-0.1719 \cos (4 s)-0.1114 \cos (6 s) .
$$

For $M=4, \lambda_{c}$ at the tenth-order of approximation has the maximum value $\left(\lambda_{c}\right)_{\max }=1.217$ at $\beta_{1}=-0.6257, \beta_{2}=-0.1727, \beta_{3}=-0.0917$ and $\beta_{4}=-0.0801$, corresponding to the optimal distribution

$$
\phi^{*}(s)=1-0.6257 \cos (2 s)-0.1727 \cos (4 s)-0.0917 \cos (6 s)-0.0801 \cos (8 s) .
$$

Obviously, if $M$ is large enough, one can get the optimal symmetrical distribution $\phi(s)$ with the property $\phi^{\prime}(0)=\phi^{\prime}(\pi)=0$. The optimal distributions $\phi(s)$ at different values of $M$ are shown in Figure 7. Note that, as the value of $M$ increases, the moments of inertia at two ends of the beam decrease and tend to zero. However, a beam with very small moment of inertia at two ends is hardly used in practice. So, the optimal distribution (3.5) of moment of inertia seems more useful in practice. Note that, for given average moment of inertia, the critical buckling load of a beam with the optimal 


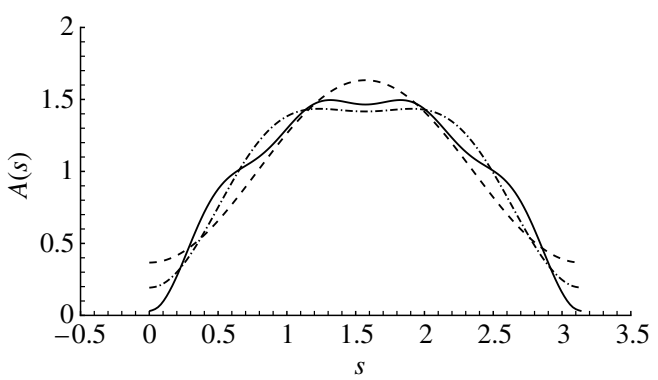

FIgurE 7. Comparison of the optimal distribution $\phi^{*}(s)$ of $I$ with the property $\phi^{\prime}(0)=0$. Dashed line, formula (3.4); dash-dotted line, formula (3.5); solid line, formula (3.6).

symmetrical distribution (3.5) of moment of inertia (with the property $\phi^{\prime}(0)=0$ ) may be about $21 \%$ larger than that of a uniform beam.

Consider the symmetrical distribution of $I$ with the property $\phi^{\prime}(0) \neq 0$. When

$$
\phi(s)=1+\beta\left[\sin (s)-\frac{2}{\pi}\right], \quad \frac{-\pi}{2} \leq s \leq \frac{\pi}{2},
$$

the tenth-order approximation of the critical axial load value reads

$$
\begin{aligned}
\lambda_{c} \approx & 1+0.212206 \beta-0.0331224 \beta^{2}-0.00240667 \beta^{3}-0.00185014 \beta^{4} \\
& -4.26374 \times 10^{-4} \beta^{5}-2.47198 \times 10^{-4} \beta^{6}-8.83328 \times 10^{-5} \beta^{7} \\
& -4.65208 \times 10^{-5} \beta^{8}-2.03725 \times 10^{-5} \beta^{9}-1.05212 \times 10^{-5} \beta^{10} \\
& -5.098152 \times 10^{-6} \beta^{11},
\end{aligned}
$$

which has maximum value $\left(\lambda_{c}\right)_{\max }=1.217$ at $\beta=\pi / 2$, corresponding to the optimal distribution

$$
\phi^{*}(s)=\frac{\pi}{2} \sin (s)
$$

For

$$
\phi(s)=1+\beta_{1}\left[\sin (s)-\frac{2}{\pi}\right]+\beta_{2}\left[\sin (3 s)-\frac{2}{3 \pi}\right],
$$

the tenth-order approximation of $\lambda_{c}$ has maximum value $\left(\lambda_{c}\right)_{\max }=1.219$ at $\beta_{1}=$ 1.5527 and $\beta_{2}=0.0543$, corresponding to the optimal distribution

$$
\phi^{*}(s)=1.5527 \sin (s)+0.0543 \sin (3 s) .
$$

Note that $\lambda_{c}$ increases by only $0.2 \%$, and the two optimal distributions are rather close, as shown in Figure 8 . Thus, $\phi^{*}(s)=(\pi / 2) \sin (s)$ should be rather close to the best distribution of moment of inertia for $\phi^{\prime}(0) \neq 0$.

Furthermore, for

$$
\phi(s)=1+\beta\left[\left(s-\frac{\pi}{2}\right)^{2}-\frac{\pi^{2}}{12}\right], \quad \frac{-6}{\pi^{2}} \leq s \leq \frac{6}{\pi^{2}},
$$




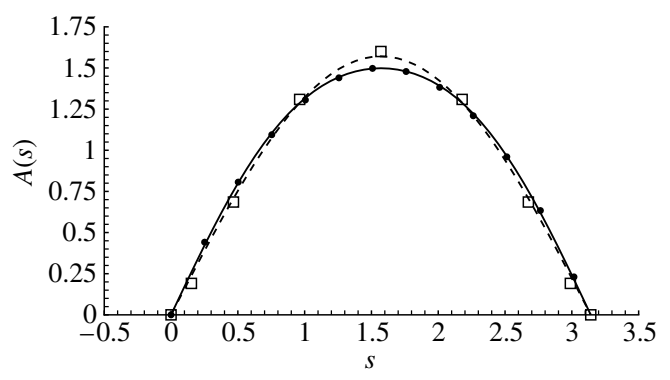

FIgURE 8. Comparison of the optimal distribution $\phi(s)$ of $I$ with the property $\phi^{\prime}(0) \neq 0$. Dashed line, formula (3.7); solid line, formula (3.8); filled symbols, formula (3.9); open symbols, formula (3.11).

TABLE 3. The value of $\lambda$ at different orders of approximation with $\hbar=-1$ for the case $\alpha=1$ and $\phi(s)=1+\beta(s-\pi / 2)$.

\begin{tabular}{ccc}
\hline & \multicolumn{2}{c}{ Calculated $\lambda$} \\
\cline { 2 - 3 } Order of approx. & $\beta=1 / 3$ & $\beta=1 / 2$ \\
\hline 2 & 1.077 & 1.018 \\
4 & 1.058 & 0.980 \\
6 & 1.050 & 0.963 \\
8 & 1.050 & 0.957 \\
$10,12,14,16,18,20$ & 1.050 & 0.956 \\
\hline
\end{tabular}

the tenth-order approximation of the critical axial load value reads

$$
\begin{aligned}
\lambda_{c}= & 1-0.5 \beta-0.164021 \beta^{2}+0.0338536 \beta^{3}-0.0551708 \beta^{4}+0.0361450 \beta^{5} \\
& -0.0472315 \beta^{6}+0.0463405 \beta^{7}-0.0582018 \beta^{8}+0.0677193 \beta^{9} \\
& -0.0864639 \beta^{10}+0.109149 \beta^{11},
\end{aligned}
$$

which has maximum value $\left(\lambda_{c}\right)_{\max }=1.218$ at $\beta=-6 / \pi^{2}$, corresponding to the optimal distribution

$$
\phi^{*}(s)=\frac{6 s(\pi-s)}{\pi^{2}} .
$$

It is interesting that the optimal distribution functions (3.8) and (3.9) of the moment of inertia are nearly the same, as shown in Figure 8. Note that the optimal distribution functions (3.7), (3.8) and (3.9) of $I$ are zero at the ends, which hardly occurs in practice. So, from a practical point of view, the optimal distribution (3.4) and (3.5) of the moment of inertia with the property $\phi^{\prime}(0)=0$ and $\phi(0)>0$ at the two ends might be more useful.

Keller [23] considered the shape of the strongest column by means of the linear buckling equation. For given volume $V$ and length $l$ of a beam whose cross sections 


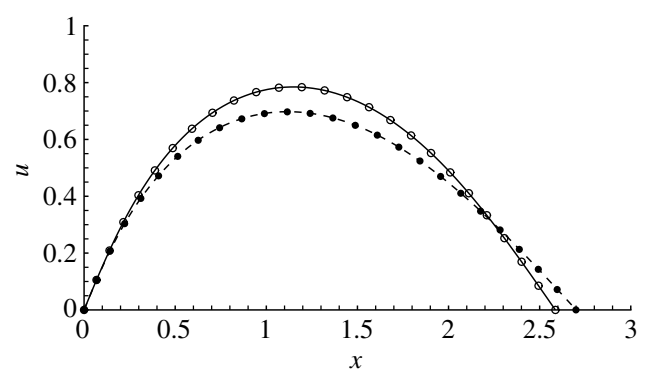

FIGURE 9. Comparison of the beam displacement at different orders of approximation when $\alpha=1$ and $\phi(s)=1+\beta(s-\pi / 2)$ with $\hbar=-1$. Solid line, tenth-order approximation when $\beta=1 / 3$; dashed line, tenth-order approximation when $\beta=1 / 2$; filled circles, 15 th-order approximation when $\beta=1 / 3$; open circles, 15 th-order approximation when $\beta=1 / 2$.

are similar, Keller [23] obtained the optimal shape of the cross section of a beam, whose area is defined by

$$
A(s)=\frac{4 V}{3 l} \sin ^{2} t, \quad s=t-\frac{1}{2} \sin (2 t), \quad t \in[0, \pi] .
$$

As pointed out by Clausen [10] and Keller [23], for given $V$ and $l$, this kind of beam can increase the critical buckling load by one third over that of a uniform one. Unlike Keller [23], we give the optimal distribution $\phi(s)$ of $I$ for given $I_{0}$, the average moment of inertia, without the assumption that all cross sections are similar. Our result indicates that, for given average moment of inertia, a beam with the optimal distribution of $I$ can increase the critical buckling load by about $22 \%$ compared to a uniform beam with the same average moment of inertia. Note that beams with the same average moment of inertia might have different volume $V$, so our results cannot be directly compared to those of Keller [23].

Keller [23] assumed that the shape of all cross sections is similar and that $I(s) \sim$ $A^{2}(s)$. Thus, for Keller's strongest beam, $\phi(s)=\mu A^{2}(s)$, where $\mu$ is a constant. On substituting (3.10) and the expression $\phi(s)=\mu A^{2}(s)$ into (1.4), we have $\mu=5 / 8$. Thus, the distribution of moment of inertia of Keller's strongest beam is given by

$$
\phi^{*}(s)=1.6 \sin ^{4}(t), \quad s=t-\frac{1}{2} \sin (2 t), \quad t \in[0, \pi] .
$$

It is interesting that, as shown in Figure 8, the above optimal distribution of moment of inertia of Keller's strongest beam is rather close to our optimal distributions (3.7), (3.8) and (3.9) of $I$. This analysis indicates that our results are consistent with those of Keller for the strongest column. Thus, for the sake of simplicity, the distribution of $I$ of the strongest beam (with $I=0$ at two ends) can be approximately expressed by a very simple formula

$$
I(\xi) \approx \frac{\pi}{2} \sin \left(\frac{\pi \xi}{l}\right)
$$




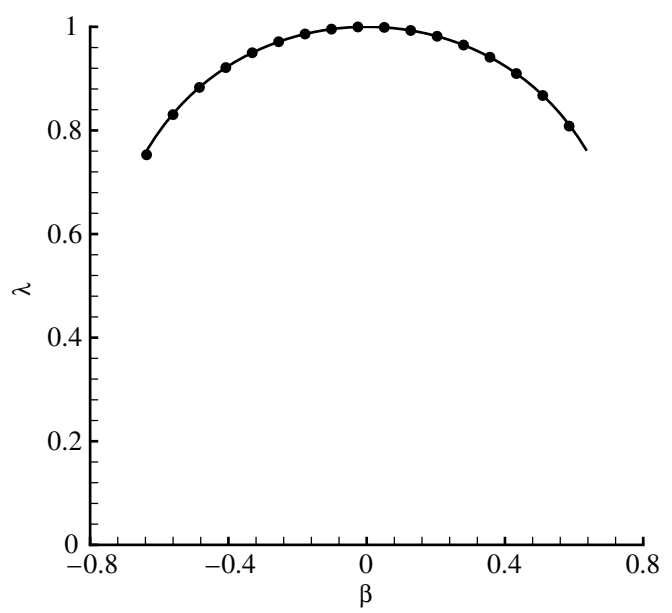

Figure 10. The critical axial load value $\lambda_{c}$ versus $\beta$ in the case of $\phi(s)=1+\beta(s-\pi / 2)$ and $\kappa=1$. Solid line, tenth-order approximation (3.12); circles, 15th-order approximation (3.13).

Finally, it should be emphasized that the optimal symmetrical distribution of moment of inertia can be found by means of the HAM. This is an advantage of the HAM over numerical techniques. It also illustrates the potential of the HAM for nonlinear eigenvalue problems governed by differential equations with variable coefficients.

3.2. Results for asymmetrical distribution of $I$ For simplicity, let us further consider the asymmetrical distribution $\phi(s)=1+\beta(s-\pi / 2)$ of the moment of inertia. In a similar way, it is found by plotting the curves $\lambda \sim \hbar$ at the tenth-order of approximation that the series of $\lambda$ is convergent when $-5 / 4 \leq \hbar \leq-1 / 4$ for all possible values of $\alpha$ and $\beta$. For example, when $\alpha=1$ and $\beta=1 / 3$ or $\beta=1 / 2$, the series solution of $\lambda$ with $\hbar=-1$ converges to 1.050 and 0.956 , respectively, as shown in Table 3. It is also found that, as long as the series of $\lambda$ converges, the corresponding series of $\theta(s)$ also converges, as shown in Figure 9. So we simply set $\hbar=-1$ here.

Physically, due to the asymmetrical distribution of $I$, the displacement $u$ of the beam is also asymmetrical, as shown in Figure 9. Mathematically, the expression for $\theta(s)$ becomes much more complicated. For example, the first- and second-order approximations of $\theta(s)$ for $\phi(s)=1+\beta(s-\pi / 2)$ are

$$
\begin{aligned}
\theta(s) \approx & \alpha\left(1-\frac{\beta s}{4}\right) \cos (s)+\frac{\alpha \beta}{4}\left(1-\pi s+s^{2}\right) \sin (s), \\
\theta(s) \approx & \alpha\left[1-\frac{s \beta}{4}-\frac{\pi}{16} \beta^{2} s\left(1-s^{2}\right)+\frac{\beta^{2}}{32} s^{2}\left(3-\pi^{2}-s^{2}\right)\right] \cos (s) \\
& +\frac{\alpha \beta}{4}\left[1+\frac{\pi \beta}{4}-\left(\pi+\frac{3 \beta}{4}+\frac{\pi^{2} \beta}{4}\right) s+(1+\pi \beta) s^{2}-\frac{3}{4} \beta s^{3}\right] \sin (s),
\end{aligned}
$$


respectively. It should be emphasized that our analytic approach is valid for a beam of arbitrary variable cross section.

As mentioned above, the critical value $\lambda_{c}$ of the axial load parameter $\lambda$ given by $\alpha=0$ has an important physical meaning. At the tenth-order approximation, $\lambda_{c}$ reads

$$
\begin{aligned}
\lambda_{c} \approx \tilde{\lambda}_{c}^{[10]} \equiv & 1-0.429350 \beta^{2}-0.219498 \beta^{4}-0.231919 \beta^{6}-0.312573 \beta^{8} \\
& -0.478608 \beta^{10}
\end{aligned}
$$

which agrees well with the 15th-order approximation

$$
\lambda_{c} \approx \tilde{\lambda}_{c}^{[15]} \equiv \tilde{\lambda}_{c}^{[10]}-0.793727 \beta^{12}-1.390927 \beta^{14}-2.538460 \beta^{16},
$$

as shown in Figure 10. Note that the critical value $\lambda_{c}$ has its maximum value $\lambda_{c}=1$ at $\beta=0$, corresponding to a beam with uniform cross section. It is found that this conclusion is valid in general. So, a uniform beam is safer than any beam with asymmetrical distribution of moment of inertia. Thus, a beam under axial load with asymmetrical distribution of moment of inertia should be avoided in practice.

\section{Conclusion and discussion}

A general analytic approach is proposed for nonlinear eigenvalue problems governed by nonlinear differential equations with variable coefficients. This approach is based on the homotopy analysis method for strongly nonlinear problems. As an example, a beam with arbitrary variable cross section acted on by a compressive axial load is used to show its validity and effectiveness. Mathematically, by means of the HAM, the original nonlinear buckling equation with variable coefficients is transformed into an infinite number of linear ODEs with constant coefficients. This greatly simplifies the solution of the original nonlinear problem. Besides, unlike perturbation techniques, such a transformation does not need any small physical parameters. More importantly, unlike all other analytic techniques, this approach introduces the so-called convergence-control parameter $\hbar$, which is an auxiliary parameter without physical meaning, to ensure the convergence of solution series so that we can always get sufficiently accurate approximations.

Physically, for arbitrary distributions of the moment of inertia, the large deflection of a beam under axial load with arbitrary variable cross section is obtained by the HAM. Different types of distributions of $I$ are investigated. It is found that a beam under axial load with symmetric olive-shaped distribution of moment of inertia is better than a uniform beam, and a uniform beam is better than a beam with symmetrical dumbbell-shaped or asymmetrical distribution of $I$. Besides, it is found that the influence of small high-frequency terms in the distribution of moment of inertia on the critical buckling load can be neglected, and therefore the small inaccuracy of $I$ due to machining precision can be neglected in practice. Note that our analytic approach is valid for a beam under axial load with arbitrary variable cross section, and provides a way to find the optimal distribution of moment of inertia. Unlike Keller [23], we search 
for the optimal distribution of moment of inertia for given average moment of inertia. Furthermore the cross section shapes need not be similar. For the distribution with the property $\phi^{\prime}(0) \neq 0$, it is found that the optimal distribution of $I$ is approximately expressed by a very simple formula

$$
\phi^{*}(\xi)=\frac{\pi}{2} \sin \left(\frac{\pi \xi}{l}\right), \quad 0 \leq \xi \leq l
$$

which can increase the critical buckling load by about $22 \%$ over that of a uniform beam with the same average moment of inertia. This conclusion is consistent with Keller's strongest column [23] for a given volume of beam. However, at the two ends, the moment of inertia of such a beam equals zero, and thus it is hardly used in practice. For the distribution with the property $\phi^{\prime}(0)=0$, it is found that the optimal distribution of $I$ is approximately expressed by

$$
\phi^{*}(\xi)=1-0.6121 \cos \left(\frac{2 \pi \xi}{l}\right)-0.1957 \cos \left(\frac{4 \pi \xi}{l}\right), \quad 0 \leq \xi \leq l,
$$

which can increase the critical buckling load by about $21 \%$ over that of a uniform beam with the same average moment of inertia. The moments of inertia of such a beam at the two ends are not zero, and thus it is more useful in practice, although its critical buckling load is about $1 \%$ less than the strongest beam with $I=0$ at two ends.

Finally, it should be emphasized that the proposed analytic approach is mathematically rather general, and thus can be applied to solve other linear/nonlinear differential equations with variable coefficients [8-18, 20-25, 36, 38, 42, 43, 45, 50].

\section{Acknowledgement}

This work is partly supported by National Natural Science Foundation of China (Approval No. 10872129) and State Key Lab of Ocean Engineering (Approval No. GKZD010002).

\section{Appendix A. Recurrence formulas of $S_{n}(s)$}

Define

$$
\begin{gathered}
S_{n}=\left.\frac{1}{n !} \frac{d^{n} \sin [q \phi(s ; q)]}{d q^{n}}\right|_{q=0}, \quad A_{n}=\left.\frac{1}{n !} \frac{d^{n} e^{i q \phi(s ; q)}}{d q^{n}}\right|_{q=0}, \\
B_{n}=\left.\frac{1}{n !} \frac{d^{n} e^{-i q \phi(s ; q)}}{d q^{n}}\right|_{q=0},
\end{gathered}
$$


where $i=\sqrt{-1}$. Then, using Euler's formula $\sin x=\left(e^{i x}-e^{-i x}\right) / 2 i$ and the definition (2.10) of $\theta_{m}(s)$, one can deduce the recurrence formulae

$$
\begin{gathered}
A_{0}=1, \quad A_{n}=i \sum_{m=0}^{n-1}\left(1-\frac{m}{n}\right) A_{m} \theta_{n-m-1}, \quad n \geq 1, \\
B_{0}=1, \quad B_{n}=-i \sum_{m=0}^{n-1}\left(1-\frac{m}{n}\right) B_{m} \theta_{n-m-1}, \quad n \geq 1, \\
S_{n}=\frac{\left(A_{n}-B_{n}\right)}{2 i}, \quad n \geq 0 .
\end{gathered}
$$

For details, refer to Liao [33].

\section{References}

[1] Z. Abbas, Y. Wang, T. Hayat and M. Oberlack, "Hydromagnetic flow in a viscoelstic fluid due to the oscillatory stretching surface", Int. Nonlinear Mech. 43 (2008) 783-793.

[2] S. Abbasbandy, "The application of the homotopy analysis method to nonlinear equations arising in heat transfer", Phys. Lett. A 360 (2006) 109-113.

[3] S. Abbasbandy, "Solitary wave equations to the Kuramoto-Sivashinsky equation by means of the homotopy analysis method", Nonlinear Dynam. 52 (2008) 35-40.

[4] S. Abbasbandy and E. J. Parkes, "Solitary smooth hump solutions of the Camassa-Holm equation by means of the homotopy analysis method", Chaos Solitons Fractals 36 (2008) 581-591.

[5] F. T. Akyildiz and K. Vajravelu, "Magnetohydrodynamic flow of a viscoelastic fluid", Phys. Lett. A 372 (2008) 3380-3384.

[6] A. Alizadeh-Pahlavan, V. Aliakbar, F. Vakili-Farahani and K. Sadeghy, "MHD flows of UCM fluids above porous stretching sheets using two-auxiliary-parameter homotopy analysis method", Commun. Nonlinear Sci. Numer. Simul. 14 (2009) 473-488.

[7] F. M. Allan, "Derivation of the Adomian decomposition method using the homotopy analysis method", Appl. Math. Comput. 190 (2007) 6-14.

[8] A. B. Boley, "On the accuracy of the Bernoulli-Euler theory for beams of variable section", J. Appl. Mech. ASME 30 (1963) 373-378.

[9] D. Chang and N. Popplewell, "A non-uniform, axially loaded Euler-Bernoulli beam having complex ends", Quart. J. Mech. Appl. Math. 49 (1996) 353-371.

[10] T. Clausen, "Über die Form architektonischer Säulen”, Bull. Phys.-Math. Acad. Imp. Sci. Saint Pétersbourg 9 (1851) 368-379.

[11] Y. A. Dugush and M. Eisenberger, "Vibrations of non-uniform continuous beams under moving loads", J. Sound Vibration 254 (2002) 911-926.

[12] I. Elishakoff and O. Rollot, "New closed-form solutions for buckling of a variable stiffness column by Mathematica", J. Sound Vibration 224 (1999) 172-182.

[13] X. Q. Fang, C. Hu and S. Y. Du, "Strain energy density of a circular cavity buried in semi-infinite functionally graded materials subjected to shear waves", Theor. Appl. Fract. Mech. 46 (2006) $166-174$.

[14] Y. Farjoun and J. Neu, "The tallest column - a dynamical system approach using a symmetry solution", Stud. Appl. Math. 115 (2005) 319-337.

[15] Y. Farjoun and J. Neu, "The optimal shape of a javelin", Stud. Appl. Math. 115 (2005) 339-354.

[16] R. D. Firouz-Abadi, H. Haddadpour and A. B. Novinzadeh, "An asymptotic solution to transverse free vibrations of variable-section beams", J. Sound Vibration 304 (2007) 530-540.

[17] M. Ganapathi, "Dynamic stability characteristics of functionally graded materials shallow spherical shells", Compos. Struct. 79 (2007) 338-343. 
[18] X. W. Gao, C. Zhang, J. Sladek and V. Sladek, "Fracture analysis of functionally graded materials by a BEM", Compos. Sci. Technol. 68 (2008) 1209-1215.

[19] P. J. Hilton, An introduction to homotopy theory (Cambridge University Press, Cambridge, 1953).

[20] X. L. Huang and H. S. Shen, "Nonlinear vibration and dynamic response of functionally graded plates in thermal environments", Internat. J. Solids Structures 41 (2004) 2403-2427.

[21] J. T. Katsikadelis and G. C. Tsiatas, "Non-linear dynamic analysis of beams with variable stiffness", J. Sound Vibration 270 (2004) 847-863.

[22] J. T. Katsikadelis and G. C. Tsiatas, "Buckling load optimization of beams", Arch. Appl. Mech. 74 (2005) 790-799.

[23] J. B. Keller, "The shape of the strongest column", Arch. Ration. Mech. Anal. 5 (1960) 275-285.

[24] B. K. Lee, J. F. Wilson and S. J. Oh, "Elastica of cantilevered beams with variable cross sections", Internat. J. Non-Linear Mech. 28 (1993) 579-589.

[25] S. K. Lee, B. R. Mace and M. J. Brennan, "Wave propagation, reflection and transmission in nonuniform one-dimensional waveguides", J. Sound Vibration 304 (2007) 31-49.

[26] S. X. Liang and D. J. Jeffrey, "Comparison of homotopy analysis method and homotopy perturbation method through an evolution equation", Commun. Nonlinear Sci. Numer. Simul. 14 (2009) 4057-4064.

[27] S. J. Liao, "On the relationship between the homotopy analysis method and Euler transform", Commun. Nonlinear Sci. Numer. Simul. 15 (2010) 1421-1431.

[28] S. J. Liao, "The proposed homotopy analysis technique for the solution of nonlinear problems", $\mathrm{Ph}$. D. Thesis, Shanghai Jiao Tong University, 1992.

[29] S. J. Liao, "A kind of approximate solution technique which does not depend upon small parameters (ii): an application in fluid mechanics", Internat. J. Non-Linear Mech. 32 (1997) 815-822.

[30] S. J. Liao, "An explicit, totally analytic approximation of Blasius viscous flow problems", Internat. J. Non-Linear Mech. 34 (1999) 759-778.

[31] S. J. Liao, Beyond perturbation-introduction to the homotopy analysis method (Chapman \& Hall/CRC Press, Boca Raton, FL, 2003).

[32] S. J. Liao, "A new branch of solutions of boundary-layer flows over an impermeable stretched plate", Int. Heat Mass Transfer 48 (2005) 2529-2539.

[33] S. J. Liao, "Notes on the homotopy analysis method: some definitions and theorems", Commun. Nonlinear Sci. Numer. Simul. 14 (2009) 983-997.

[34] S. J. Liao and E. Magyari, "Exponentially decaying boundary layers as limiting cases of families of algebraically decaying ones", Z. Angew. Math. Phys. 57 (2006) 777-792.

[35] S. J. Liao and Y. Tan, "A general approach to obtain series solutions of nonlinear differential equations", Stud. Appl. Math. 119 (2007) 297-355.

[36] L. Marin, "Numerical solution of the Cauchy problem for steady-state heat transfer in twodimensional functionally graded materials", Internat. J. Solids Structures 42 (2005) 4338-4351.

[37] A. Molabahrami and F. Khani, "The homotopy analysis method to solve the Burgers-Huxley equation", Nonlinear Anal. B 10 (2009) 589-600.

[38] V. G. Paranicolaou, "The periodic Euler-Bernoulli equation", Trans. Amer. Math. Soc. 355 (2003) $3727-3759$.

[39] H. Poincaré, "Second complément à l'analysis situs", Proc. London Math. Soc. 32 (1900) 277-308.

[40] M. Sajid and T. Hayat, "Comparison of HAM and HPM methods for nonlinear heat conduction and convection equations", Nonlinear Anal. B 9 (2008) 2296-2301.

[41] M. Sajid, T. Hayat and S. Asghar, "Comparison between the HAM and HPM solutions of thin film flows of non-Newtonian fluids on a moving belt", Nonlinear Dynam. 50 (2007) 27-35.

[42] E. J. Sapountzakis and G. C. Tsiatas, "Elastic flexural buckling analysis of composite beams of variable cross-section by BEM", Eng. Struct. 29 (2007) 675-681. 
[43] H. S. Shen, "Nonlinear bending response of functionally graded plates subjected to transverse loads and in thermal environments", Int. J. Mech. Sci. 44 (2002) 561-584.

[44] H. Song and L. Tao, "Homotopy analysis of 1D unsteady, nonlinear groundwater flow through porous media", J. Coastal Res. 50 (2007) 292-295.

[45] B. L. Wang and Y. W. Mai, "A periodic array of cracks in functional graded materials subjected to thermo-mechanical loading", Internat. J. Engrg. Sci. 43 (2005) 432-446.

[46] Y. Y. Wu, "Analytic solutions for nonlinear long wave propagation", Ph. D. Thesis, University of Hawaii at Manoa, 2009.

[47] Y. Y. Wu and K. F. Cheung, "Explicit solution to the exact Riemann problems and application in nonlinear shallow water equations", Internat. J. Numer. Methods Fluids 57 (2008) 1649-1668.

[48] Y. Y. Wu and K. F. Cheung, "Homotopy solution for nonlinear differential equations in wave propagation problems", Wave Motion 46 (2009) 1-14.

[49] K. Yabushita, M. Yamashita and K. Tsuboi, "An analytic solution of projectile motion with the quadratic resistance law using the homotopy analysis method", J. Phys. A 40 (2007) 8403-8416.

[50] J. Yang and H. S. Shen, "Non-linear analysis of functionally graded plates under transverse and in-plane loads", Internat. J. Non-Linear Mech. 38 (2003) 467-482.

[51] S.-P. Zhu, "A closed-form analytical solution for the valuation of convertible bonds with constant dividend yield", ANZIAM J. 47 (2006) 477-494.

[52] L. Zou, Z. Zong, Z. Wang and L. He, "Solving the discrete KdV equation with homotopy analysis method", Phys. Lett. A 370 (2007) 287-294. 